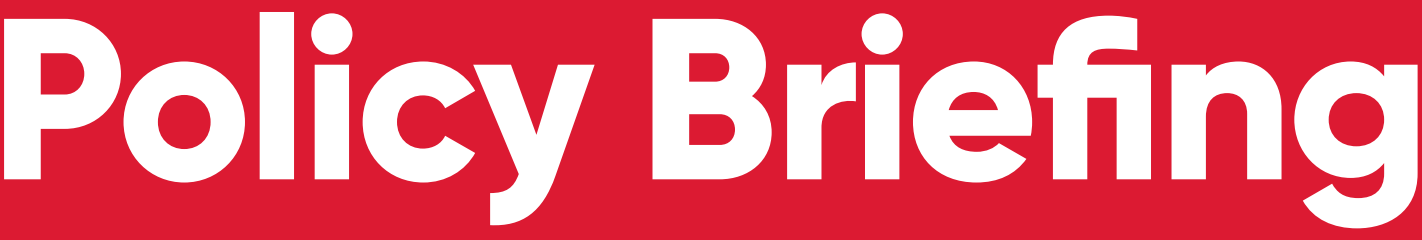

\title{
Avenir imaginé : insuffisan
du soutien aux jeunes en milieu rural en Côte d'Ivoire
}

Le manque à des débouchés professionnels pour les jeunes est une préoccupation majeure en Afrique, et a un impact sur de nombreux domaines stratégiques. En Côte d'Ivoire, la situation est difficile en raison des crises politiques et économiques qui ont frappé le pays au cours des dernières décennies et qui ont eu un impact négatif sur l'éducation et accentué la précarité de l'emploi, en particulier pour la jeunesse rurale. Afin de s'attaquer au problème dans les zones rurales, il faut une approche centrée sur les jeunes qui met en place des interventions à long terme, bien ciblées, coordonnées et basées sur les réalités des jeunes.

\section{Messages clés}

- Les jeunes en milieu rural de Côte d'Ivoire aspirent à la stabilité financière et à une position sociale au sein de leur communauté. Malgré l'inégalité des sexes, les jeunes, quel que soit leur sexe, souhaitent acquérir des biens matériels, comme une maison et des biens domestiques, et pouvoir également apporter un soutien matériel aux membres de leur famille élargie.

- Les jeunes en milieu rural ont une vision pragmatique de leurs possibilités d'emploi. Leurs projets reflètent une évaluation réfléchie du type d'activités rémunératrices dans lesquelles ils devront s'investir pour subvenir à leurs propres besoins et à ceux de leurs familles.

- En s'appuyant sur les connaissances des jeunes, on peut retenir que l'amélioration de la formation professionnelle, de l'accès à des prêts bancaires et à des transferts d'espèces permet de les soutenir afin d'élargir leur participation dans les chaînes de valeur régionales et mondiales, tout en tenant compte de l'égalité des sexes.

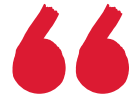

Un grand nombre de jeunes en milieu rural ont des responsabilités importantes en tant que conjoints, parents et pourvoyeurs. Ils souhaitent une vie moins précaire. 


\section{Défis de la jeunesse rurale}

À la fin de la décennie 2009-2018 de l'Union africaine pour le développement de la jeunesse, la Fondation pour le renforcement des capacités en Afrique a organisé un sommet pour identifier des solutions innovantes au défi persistant de la création d'emplois décents sur le continent africain. Quatre suggestions clés ont mis en évidence la nécessité de :

- Transformer le secteur agricole pour intégrer les petites et moyennes entreprises (PME) dans les chaînes de valeur régionales et mondiales ;

- Développer l'agro-industrie locale pour apporter une valeur ajoutée ;

- Faciliter l'accès au capital d'investissement pour les PME ; et

- Changer l'orientation de l'enseignement pour promouvoir la formation aux emplois techniques et professionnels.

Pour parvenir à ces transformations, il faut une approche centrée sur les jeunes qui met en place des interventions à long terme, bien ciblées, coordonnées et basées sur les réalités rurales.

En Côte d'Ivoire, la question du travail décent est cruciale, principalement en raison des conflits politiques et économiques de l'histoire récente du pays qui ont eu un impact négatif sur l'éducation et accentué la précarité de l'emploi, en particulier chez les jeunes. La Banque mondiale note que $45,8 \%$ des jeunes entre 15 et 34 ans étaient économiquement actifs en 2014, avec des écarts de $28,1 \%$ en milieu urbain et de $64,8 \%$ en milieu rural.

La participation plus faible des jeunes en milieu urbain aux activités économiques pourrait expliquer pourquoi la plupart des interventions en faveur de l'emploi des jeunes concernaient principalement les zones urbaines. Cependant, en 2014, seulement $11,1 \%$ de la population rurale avaient un travail salarié, alors que $88,9 \%$ travaillaient dans de petites entreprises indépendantes ou familiales caractérisées par une faible productivité et un sous-emploi. En 2015, plus de la moitié de la population rurale vivait en dessous du seuil national de paurreté de 737 francs CFA (1,23 USD) par jour, ce qui souligne l'importance d'accroître les possibilités de travail décent pour les jeunes ruraux.

Ce rapport de synthèse est basé sur une recherche menée en Côte d'Ivoire qui a exploré les défis et les possibilités d'emploi des jeunes en milieu rural dans le cadre d'un programme de recherche comparative financé par le Fonds international de développement agricole (FIDA). Il a utilisé une méthodologie mixte pour étudier l'engagement des jeunes dans l'économie rurale, leurs aspirations pour l'avenir et l'impact de l'éducation.

La recherche s'est déroulée dans les régions de Daloa, Soubré, Odienné et Korhogo, et a fait appel à des méthodes qualitatives et participatives pour explorer les activités de subsistance agricoles et non agricoles entreprises par les jeunes de 15 à 30 ans. Un petit nombre de jeunes femmes et hommes ont pris des photos pour illustrer leurs aspirations, ce qui a facilité les discussions sur la manière dont les jeunes cherchent à suivre les chemins de la réussite. Des entretiens de groupe et individuels avec des jeunes et des adultes plus âgés ont permis de mieux comprendre le contexte de la vie des jeunes au sein de chaque communauté.

Ce rapport de synthèse présente les perspectives des jeunes ruraux dans trois domaines: (1) leur avenir imaginé ; (2) les différentes possibilités d'emploi ; et (3) les obstacles à la concrétisation de leurs aspirations. Nous analysons les données recueillies dans les quatre régions en relation avec les quatre suggestions clés présentées ci-dessus pour créer du travail et de meilleurs revenus.

\section{Avenir imaginé}

Dans nos sites de recherche, les jeunes imaginent leur avenir avec moins de pauvreté et de précarité. Nombre d'entre eux ont 
des responsabilités importantes en tant que conjoints, parents et pourvoyeurs. Leur vision de l'avenir est principalement rurale ou implique des petites villes ayant un potentiel de génération de revenus non agricoles. Très peu rêvent de voyager plus loin.

Nos discussions ont tourné autour de ce que les jeunes pensaient être capables de faire, si leur situation était différente. Les scénarios ont fourni beaucoup d'informations sur l'inégalité des sexes et l'effet sur les jeunes des contingences dans leur famille. Une femme mariée de 21 ans avec deux enfants aurait aimé devenir médecin. Si elle avait été de sexe masculin, elle aurait eu plus de chances d'être inscrite à l'école. Des jeunes hommes ayant abandonné l'école en raison de la pauvreté, de la perte d'un parent ou d'une maladie ont également parlé du type de professions plus intéressantes auxquelles l'éducation aurait pu leur permettre d'avoir accès ou de la migration vers l'Europe.

Toutefois, ils ont tous en commun une vision pragmatique de ce qui est exigé d'eux dans leur situation actuelle et à l'avenir.

Presque toutes les familles sont impliquées dans l'agriculture et certains jeunes envisagent de réussir en se diversifiant dans de nouveaux produits, tels que l'anarcade et d'autres cultures pérennes. Un agriculteur de 30 ans d'Odienné espérait étendre et moderniser son exploitation en acquérant d'abord plus de terres et de bétail, puis en utilisant des machines et des produits agricoles pour lutter contre les parasites et les maladies. Il a également envisagé la possibilité de créer une entreprise de transformation. À la fin, il rêvait de devenir ingénieur agronome. Les jeunes se voyant agriculteurs ont tendance à approuver les changements visant à accroître la productivité agricole, mais leurs idées sont basées sur les petites exploitations agricoles plutôt que sur des transformations structurelles radicales.

Si l'idée de diriger une entreprise pour améliorer sa perspective d'avenir est aussi courante chez les jeunes hommes que les

\section{Les jeunes en milieu rural planifient des activités génératrices de revenus basées sur une évaluation pragmatique de ce dont ils ont besoin pour subvenir à leurs besoins et à ceux de leur famille.}

jeunes femmes, le choix du produit ou de l'activité est généralement sexospécifique. Les jeunes hommes souhaitent travailler comme mécaniciens, maçons ou menuisiers, ou dans la réparation d'appareils électriques, tandis que les jeunes femmes veulent développer le commerce d'articles alimentaires ou de vêtements, ou ouvrir un salon de coiffure. Beaucoup associent les activités non agricoles à un statut social plus élevé, mais imaginent malgré tout cultiver des aliments pour la consommation de leur famille, à moins qu'ils ne s'installent dans une ville ou une petite ville rurale. Leurs aspirations s'appuient sur des activités qu'ils pratiquent déjà. Ils sont pragmatiques et rêvent rarement d'être patrons.

La plupart des jeunes ruraux aspirent à la réussite sociale, ce qui pour eux équivaut à la stabilité financière, à la capacité d'assurer le bien-être de leur famille et à atteindre une position sociale au sein de leur communauté. La réussite est marquée par la possession de certains biens matériels, comme une maison, mais elle renvoie également à la capacité d'aider les membres de leur famille. La réussite dépend également de conditions de vie plus agréables grâce, entre autres, à la possession de son propre véhicule et l'achat de biens domestiques, tels que des réfrigérateurs ou des meubles.

\section{Débouchés professionnels}

Les jeunes des zones rurales de Côte d'Ivoire s'impliquent dans diverses activités économiques. Ils ont généralement acquis des compétences agricoles dès leur plus 
jeune âge en travaillant avec leurs parents en dehors des heures de classe et pendant les vacances scolaires. Les compétences acquises dans l'enfance sont essentielles plus tard dans la vie. Une femme de 20 ans vivant près de Korhogo a expliqué comment elle a commencé à cultiver les mêmes légumes que sa mère avait cultivés avant elle, car elle savait déjà comment faire. Les jeunes hommes utilisent également les compétences acquises auprès de leurs parents. Un homme de 22 ans, près de Soubré, a expliqué comment il avait appris à cultiver l'igname et le maïs en travaillant avec son père, et le gombo et l'aubergine en travaillant avec sa mère. D'autres jeunes hommes ont acquis des compétences pour cultiver des cultures de rente comme le cacao, le café, le caoutchouc, l'anacarde ou le coton.

Les possibilités d'emploi dans l'agriculture sont un mélange de travail familial non rémunéré et de travail sur sa propre ferme. Certains jeunes hommes acceptent un travail agricole occasionnel pendant quelques mois afin de gagner un revenu supplémentaire lorsque leur travail n'est pas nécessaire à la maison. Les jeunes femmes travaillent dans l'agriculture avec leur mère et, après leur mariage, avec leur mari ou leur belle-mère. Les jeunes femmes mariées peuvent avoir leur propre petite exploitation agricole ou effectuer des travaux agricoles occasionnels si la famille est très pauvre.

La plupart des jeunes agriculteurs font les mêmes cultures que leurs parents. Mais en raison de la baisse des rendements et de la

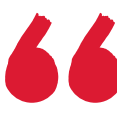

\section{Les jeunes hommes} approuvent les changements agricoles, mais leurs idées sont basées sur les petites exploitations agricoles plutôt que sur des transformations structurelles radicales. fluctuation des prix, l'intérêt pour l'expansion des cultures commerciales traditionnelles est moindre. Les jeunes hommes se tournent vers la culture d'anacarde ou de riz s'ils peuvent accéder à des terres, tandis que les jeunes femmes cultivent plutôt des légumes pour le marché. Parfois, certains adoptent de nouvelles variétés ou méthodes de culture. Un homme de 29 ans dans les environs de Korhogo a décrit comment il apprenait à faire la culture de tomates hors sol (hydroponique). Un petit nombre de jeunes hommes se consacrent également à l'élevage microcommercial d'animaux pour obtenir de meilleurs revenus.

Les jeunes s'intéressent de plus en plus aux activités génératrices de revenus non agricoles, comme l'achat de cultures commerciales, le commerce sur les marchés locaux et les métiers tels que la couture, la coiffure, la menuiserie, la maçonnerie ou la mécanique. Les jeunes hommes entrent dans les chaînes de valeur du cacao et d'anacarde en tant qu'acheteurs à la ferme, comme l'explique un homme de 28 ans de la région de Korhogo :

Quand la saison des noix de cajou commence, je vais dans les petits villages et campements pour acheter leur anacarde. Les agriculteurs me téléphonent lorsqu'ils ont des récoltes à vendre. Je travaille avec un commerçant de Korhogo qui me donne le prix de l'anacarde par téléphone et quand j'en ai acheté suffisamment, je les lui revends. Quand la récolte d'anacarde est terminée, je vends des produits agricoles tels que l'engrais et des pesticides.

Les jeunes femmes entrant dans les chaînes de valeur achètent des produits cultivés principalement par des femmes, comme le gombo et le poivron. Des jeunes femmes travaillent à la transformation du manioc pour en faire de l'attiéké, un aliment de base en Côte d'Ivoire. Cependant, les processus de valorisation consistent essentiellement à préparer des aliments à vendre. 
L'intérêt pour le commerce est énorme chez les jeunes, que ce soient les hommes ou les femmes. Cependant, comme les femmes ont moins accès à la terre, elles dépendent davantage des activités non agricoles.

Deux femmes de 28 ans vivants près de Daloa décrivent une trajectoire commune. L'une d'entre elles voulait se lancer dans le commerce, mais prévoyait de commencer petit à petit et si elle gagnait suffisamment, elle ouvrirait un magasin. L'autre était déjà commerçante de dentelles et robes. Elle espérait ouvrir un magasin sur le marché, mais pense que cela ne serait peut-être pas réalisable, du moins à moyen terme.

Enfin, un grand nombre de jeunes combinent le travail agricole et non agricole. Dans la région d'Odienné, des jeunes travaillent dans l'extraction artisanale de l'or. Dans tous les sites de l'étude, de jeunes hommes se lancent dans des travaux de construction non qualifiés, acquérant progressivement des compétences en maçonnerie, en menuiserie et en décoration. Les jeunes femmes se lancent surtout dans le commerce. D'autres, qui ont été contraints d'interrompre leurs études secondaires, font des apprentissages artisanaux en vue de créer un atelier à l'avenir.

\section{Obstacles à une vie décente}

Quelle que soit la région, la question de la rareté des terres a été soulevée dans la plupart des entretiens individuels et de groupe comme un problème grave étant donné que les activités productives dans les zones rurales sont essentiellement agricoles. Notre étude indique que les dynamiques engendrées par la rareté des terres varient. À Daloa, la principale zone de production de cacao en Côte d'Ivoire jusqu'aux années 1980, les champs de cacao sont de moins en moins productifs, car les sols s'appauvrissent de plus en plus au fil des décennies. Les cultivateurs de cacao se tournent vers la production de cultures vivrières, mais principalement comme moyen de subsistance. Leur capacité réduite à

\section{Les jeunes femmes accédant aux chaînes de valeur mettent l'accent sur les cultures vivrières : les opportunités d'apprendre les techniques de transformation dans le village sont rares.}

générer des revenus limite également leur capacité à fournir des opportunités d'emploi pour les jeunes. À Soubré, une zone de production importante pour les cultures d'exportation, la vente des terres par les propriétaires rend difficile pour les jeunes hommes et presque impossible pour les jeunes femmes d'obtenir des terres pour se lancer dans des cultures purement commerciales.

À Odienné et Korhogo, le régime foncier est plus traditionnel et lié à l'organisation de la lignée. Lorsque les jeunes ont accès à la terre, les conditions d'accès sont parfois compliquées et incertaines. Un homme de 28 ans de la région de Korhogo a expliqué que la terre qu'il cultive avec son frère aîné n'appartient à aucun des deux. Leur père l'a pris en charge à la mort de son frère parce les enfants de celui-ci étaient trop jeunes pour la cultiver eux-mêmes. À la mort de leur père, leurs cousins pourraient revendiquer leur droit à la terre.

La fixation des prix sur le marché mondial affecte la viabilité des cultures commerciales dans les quatre sites de recherche. Même lorsque ces cultures perdent leur valeur commerciale, les agriculteurs continuent souvent à les cultiver parce qu'ils obtiennent des engrais et des pesticides par I'intermédiaire des coopératives d'achat et des entreprises, qu'ils utilisent ensuite pour les cultures vivrières. Les jeunes hommes hésitent à s'engager dans la production de cultures à faibles revenus, mais ils manquent de soutien institutionnel pour se diversifier. 
En général, la transformation des produits agricoles reste limitée au niveau du village, ce qui constitue un obstacle important, en particulier pour les jeunes femmes. Nos données indiquent que les femmes plus âgées cultivent des cultures vivrières et s'engagent dans différentes formes de commerce. Les jeunes femmes ont peu d'occasions d'apprendre les techniques de culture de transformation, à moins qu'elles ne séjournent chez des membres de la famille impliqués dans une telle activité. Les possibilités des jeunes de prospérer dans le secteur agricole diminuent par conséquent, et il est de plus en plus nécessaire de soutenir des activités génératrices de revenus non agricoles.

La taille de la plupart des entreprises rurales constitue une contrainte majeure. Beaucoup sont si petites qu'elles dépendent uniquement du propriétaire, du travail familial non rémunéré ou de très peu d'employés. De ce fait, la plupart des jeunes dépendent de la création de leur propre microentreprise. La stratégie la plus courante utilisée par les jeunes, hommes et femmes, pour obtenir un capital de démarrage consiste à constituer une épargne en lançant de petites activités locales à faible coût. Une autre stratégie consiste à espérer que quelqu'un leur donne le capital nécessaire. Dans le cas des jeunes femmes, le mari peut fournir l'argent nécessaire, et les jeunes des deux sexes peuvent recevoir l'aide de membres de la famille. La plupart hésitent à contracter un prêt de manière formelle par crainte de ne pas pouvoir le rembourser.

L'épargne des jeunes est limitée et souvent soumise à des demandes concurrentes. Une femme divorcée de 30 ans, près de Daloa, a décrit comment elle avait gagné 190000 FCFA (315 USD) la première année où elle a récolté de l'anacarde, mais seulement 30000 FCFA (50 USD) la troisième année parce qu'elle est tombée malade. Elle a eu du mal à investir dans son entreprise, car elle avait besoin d'argent pour payer les funérailles de sa mère et plus tard des frais médicaux. Des demandes concurrentes peuvent donc saper les plans rémunérateurs et frapper plus durement les jeunes en raison de leurs responsabilités en matière de soins aux jeunes enfants et aux parents vieillissants.

Une autre contrainte est le faible niveau d'éducation. De nombreux enfants et jeunes sont fatigués pendant les cours ou n'ont pas assez de temps pour réviser à la maison parce qu'ils aident leurs parents. Le travail peut avoir un impact négatif sur les résultats scolaires des jeunes, mais le manque de finances est la principale raison de l'abandon scolaire. Cependant, le travail peut également contribuer à les maintenir à l'école lorsqu'il leur permet de payer eux-mêmes une partie des dépenses scolaires.

Notre enquête montre que ceux qui restent plus longtemps dans le système éducatif sont plus susceptibles de se diversifier dans de nouvelles activités génératrices de revenus ou de suivre une formation professionnelle informelle. Afin de développer une économie rurale plus dynamique, il est donc important de soutenir activement l'enseignement scolaire et la formation professionnelle, surtout pour ceux qui risquent de quitter l'école plus tôt.

Les programmes nationaux existants visant à améliorer l'accès à l'emploi ont des limites. Ces programmes ont tendance à mettre l'accent sur la formation des compétences, sans reconnaître les compétences et les connaissances informelles dont sont susceptibles de déjà disposer les jeunes ruraux. Ou bien ils promeuvent des programmes de microfinancement à court terme sans tenir compte des multiples responsabilités des jeunes ruraux, ce qui nécessite parfois des compromis.

De la même manière, les transformations du secteur agricole visant à intégrer les PME dans les chaînes de valeur régionales et mondiales ne créent pas automatiquement des emplois pour les jeunes ruraux, hommes et femmes.

\section{ids.ac.uk}




\section{Recommandations en matière de politique}

L'accès à la terre, à la main d'œuvre et à d'autres ressources étant très inégalitaire et sexospécifique, il est impératif de concevoir des programmes tenant compte de l'égalité des sexes. Les programmes doivent veiller à ce que les jeunes hommes et les jeunes femmes bénéficient d'un soutien équitable pour se développer dans des domaines de travail qui sont perçus comme culturellement appropriés, avec une certaine marge de manœuvre pour remettre en question les normes conventionnelles, si nécessaire.

- Les cultures, les pratiques culturales et le potentiel d'intensification doivent tous être pris en considération. Compte tenu de la demande croissante de riz, par exemple, l'Office national pour le développement de la riziculture (ONDR) a piloté un projet visant à développer la chaîne de valeur locale afin d'accroître la production et de créer de nouveaux emplois non agricoles.

- Le développement de la chaîne de valeur du riz doit prendre en considération comment intégrer les jeunes hommes à différents niveaux. Notre étude montre l'importance pour les jeunes hommes de l'achat à la ferme comme point d'entrée principale dans les chaînes de valeur des cultures d'exportation. Il est peu probable qu'ils puissent créer des emplois pour eux-mêmes et pour d'autres dans les chaînes de valeur en raison de la taille réduite de leurs entreprises et du manque de capital.
- Un plus grand investissement est nécessaire pour développer des chaînes de valeur des légumes et du manioc qui sont des domaines essentiellement féminins. La production de l'attiéké reste familiale et peu qualifiée, mais la chaîne de valeur crée néanmoins des possibilités pour les femmes d'entrer dans le secteur commercial.

- Pour élargir la participation à un plus grand nombre de jeunes femmes, la formation aux techniques de transformation pourrait être développée en dehors de la famille, éventuellement par une professionnalisation de la production pour créer des emplois et réduire la dépendance sur le travail familial non rémunéré.

- L'accès au capital doit prendre en compte les préoccupations et les responsabilités des jeunes quant aux conditions de remboursement des prêts. Comme la moitié environ de la population rurale vit dans la pauvreté, les transferts en espèce du type filet social pourraient constituer une meilleure option que la microfinance.

- Des formations professionnelles à plus long terme sont nécessaires pour créer des débouchés professionnels et entrepreneuriaux. Notre étude montre que l'enseignement technique et la formation professionnelle sont importants. Toutefois, de nombreux programmes existants ne proposent que des formations courtes sur un ensemble limité de compétences. Nous avons constaté que tous ceux qui avaient un métier et qui aspiraient à agrandir leur activité avaient suivi un apprentissage informel. 
Institute of Development Studies, Library Road, Brighton, BN1 9RE, Royaume-Uni +44 (0)1273 606261 ids.ac.uk

IDS est une organisation à but non lucratif limitée par garantie et enregistrée en Angleterre. Numéro d'association caritative 306371. Numéro d'organisation à but non lucratif 877338.

\section{Suggestions de lecture supplémentaire}

ACBF (2018) Lutte contre le chômage des jeunes en

Afrique : solutions innovantes proposées par les think tanks, Rapport du $5^{\text {ème }}$ sommet des Think Tanks d'Afrique, Harare : Fondation pour le renforcement des capacités en Afrique

Christiaensen, L. et Premand, P. (2017) Vers de meilleurs emplois et l'inclusion productive : Emploi, Productivité et Inclusion pour Réduire la Pauvreté : Un diagnostic de la situation de l'emploi en Côte d'Ivoire, Washington DC : Groupe de la Banque Mondiale INS (2015) Enquête sur le niveau de vie des ménages e Côte d'Ivoire (env 2015), Profil de pauvreté, Abidjan: Institut National de la Statistique

Mendez del Villar, P. et coll. (2017) Analyse de la chaîne de manioc en Côte d'Ivoire, Rapport pour I'Union Européenne

Sumberg, J. (ed.) (2021) Youth and the Rural Economy in Africa: Hard Work and Hazard, Wallingford: CABI (Accès libre)
Ce rapport IDS a été rédigé par Affoué Philomène Koffi (Université Félix Houphouët-Boigny) et Dorte Thorsen (IDS), et édité par Carol Smithyes (IDS). Il a été élaboré dans le cadre du programme de recherche financé par le FIDA intitulé " Challenges and Opportunities for Rural Youth Employment in Sub-Saharan Africa: A Mixed-Methods Study to Inform Policy and Programmes " (en français, Défis et opportunités de l'emploi de la jeunesse rurale en Afrique subsaharienne : étude multiméthodologique visant à renseigner les politiques et les programmes).

Les opinions exprimées dans cette publication sont celles des auteurs et ne reflètent pas nécessairement celles d'IDS ou du FIDA, ou leurs politiques.

(c) Institute of Development Studies 2021.

(c) (i) (-) Ce rapport est d'accès libre et est ${ }_{\mathbf{B Y}} \mathbf{N a}$ distribué selon les termes de la licence Creative Commons Attribution Non Commercial 4.0 International licence (CC BY-NC), qui permet I'utilisation, la distribution et la reproduction de tout support d'information pourvu que le mérite de sa création soit imputé à ses auteurs et à sa source, que toute modification ou adaptation soit indiqué, et qu'il ne soit pas utilisé à des fins commerciales. ISSN 1479-974X

DOI: 10.19088/IDS.2021.042 